This article has been downloaded from IOPscience. Please scroll down to see the full text article. (http://iopscience.iop.org/0031-9155/53/1/010)

More related content is available

Download details:

IP Address: 129.59.92.115

The article was downloaded on 18/01/2008 at 15:52

Please note that terms and conditions apply. 


\title{
Evaluation of 3D modality-independent elastography for breast imaging: a simulation study
}

\author{
J J Ou ${ }^{1}$, R E Ong ${ }^{1}$, T E Yankeelov ${ }^{1,2,3,4}$ and M I Miga ${ }^{1,2,3}$ \\ ${ }^{1}$ Department of Biomedical Engineering, Vanderbilt University, Nashville, TN 37235, USA \\ 2 Department of Radiology and Radiological Sciences, Vanderbilt University Medical Center, \\ Nashville, TN 37232, USA \\ 3 Vanderbilt University Institute of Imaging Science, Nashville, TN 37232, USA \\ ${ }^{4}$ Department of Physics and Astronomy, Vanderbilt University, Nashville, TN 37235, USA \\ E-mail: jao.ou@vanderbilt.edu and michael.i.miga@vanderbilt.edu
}

Received 26 April 2007, in final form 1 November 2007

Published 19 December 2007

Online at stacks.iop.org/PMB/53/147

\begin{abstract}
This paper reports on the development and preliminary testing of a threedimensional implementation of an inverse problem technique for extracting soft-tissue elasticity information via non-rigid model-based image registration. The modality-independent elastography (MIE) algorithm adjusts the elastic properties of a biomechanical model to achieve maximal similarity between images acquired under different states of static loading. A series of simulation experiments with clinical image sets of human breasts were performed to test the ability of the method to identify and characterize a radiographically occult stiff lesion. Because boundary conditions are a critical input to the algorithm, a comparison of three methods for semi-automated surface point correspondence was conducted in the context of systematic and randomized noise processes. The results illustrate that 3D MIE was able to successfully reconstruct elasticity images using data obtained from both magnetic resonance and $\mathrm{x}$-ray computed tomography systems. The lesion was localized correctly in all cases and its relative elasticity found to be reasonably close to the true values $(3.5 \%$ with the use of spatial priors and $11.6 \%$ without). In addition, the inaccuracies of surface registration performed with thin-plate spline interpolation did not exceed empiric thresholds of unacceptable boundary condition error.
\end{abstract}

\section{Introduction}

Breast cancer is the most common cancer of women in the United States, the second most common cause of cancer death in women and the leading cause of death in women aged 45 to 55. Estimates for the year 2007 indicate that 178480 American women will be diagnosed with the disease and 40910 women will die from it (ACS 2007). While many advances have been made in the treatment of the disease, the ability to detect its presence for either screening 
or diagnostic purposes remains an area of active research involving many novel forms of imaging. The characterization of the mechanical properties of breast tissue is an important potential source of clinical information because of the long-standing association of palpable differences in stiffness with possible pathological states. A minimally invasive methodology for analyzing tissue elasticity through imaging and/or image processing techniques is a central goal of the field of elastography (Parker et al 2005), with the application of various techniques being found not only in the interrogation of the breast (McKnight et al 2002, Melodelima et al 2006, Sinkus et al 2000), but also in skin (Miga et al 2005, Tsap et al 1998, Zhang et al 2004), prostate (Curiel et al 2005, Egorov et al 2006) and other accessible organ systems.

Many of the current elastography methods are founded in ultrasound (US) (Ophir et al 1991, 2000) and magnetic resonance (MR) (Manduca et al 2001, Muthupillai et al 1995) imaging and involve the estimation of induced displacements within the tissue of interest to infer the elasticity distribution. We have recast the problem as a physically constrained, nonrigid image registration utilizing numerical models of static deformation with image similarity metrics to reconstruct the spatial distribution of elasticity parameters. This technique has been termed 'modality-independent elastography' (MIE) (Miga 2002, 2003, Washington and Miga 2004) because of its ability to handle anatomical images from different sources with relatively simple modifications to the acquisition procedure. To date, data from MR, x-ray computed tomography (CT) and digital photography have been used to successfully drive the algorithm in two-dimensional (2D) work. Others have also pursued similar approaches within the context of ultrasound elastography (Garra et al 1997, Gokhale et al 2004, Sarvazyan et al 1995), optical image analysis (Tsap et al 1998) and to a lesser extent with magnetic resonance elastography (Fowlkes et al 1995). While the use of MIE in 2D has been illuminating for algorithmic development and may have its own applications in studying the more planar system of the skin, ultimately, translation of the method to utilize volumetric data is desirable (if not necessary) in order to provide an accurate representation of organs such as the breast as a whole. In this work, we present a newly realized three-dimensional (3D) version of MIE along with simulation experiments to evaluate its performance. In addition, some potential effects of degraded input quality are addressed by examining robustness of the algorithm to inaccuracies under specified boundary conditions and then comparing the reconstruction fidelity of three different techniques developed for semi-automatic generation of boundary conditions.

\section{Methods and materials}

\subsection{MIE reconstruction framework}

The conceptual framework for our elastographic reconstruction has been previously described in Miga (2002, 2003), Miga et al (2005) and Washington and Miga (2004)). To review, an image of a tissue of interest (source) is deformed by a biomechanical model and compared against an acquired image of the same tissue in a mechanically loaded state (target). Iterative updates of elasticity parameters to the model are performed until a suitable match in image similarity is achieved in a least-squares manner to satisfy a nonlinear optimization scheme. This process as illustrated in figure 1 can be classified as an inverse problem, with model-based deformation and registration of the source image representing the forward problem.

The three major components of the reconstruction algorithm are the biomechanical model, image comparison and the optimization routine. Although there are a number of models for soft-tissue mechanics, it is reasonably appropriate to begin with a general elastic body. The partial differential equation (PDEs) that expresses a state of mechanical equilibrium is

$$
\nabla \cdot \sigma=0
$$




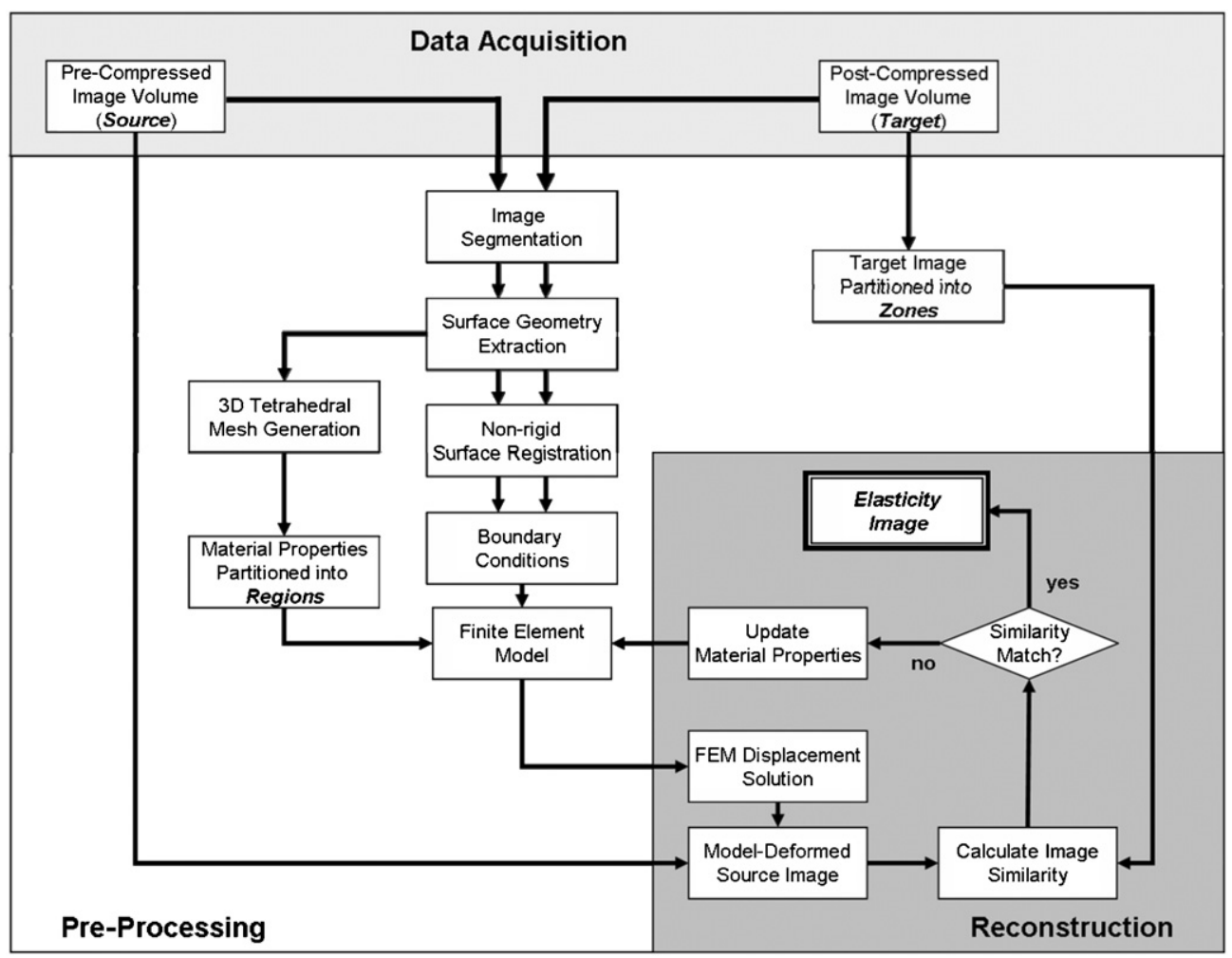

Figure 1. Schematic of MIE framework. After acquisition of image data, surface representations are segmented from the pre- and post-deformation volumes (source and target, respectively). A number of pre-processing steps are performed to generate boundary conditions for the biomechanical model, which produces a deformed image that can be compared with the true target volume. The optimization routine updates the elasticity distribution until the best similarity is achieved.

where $\sigma$ is the Cartesian stress tensor (Boresi and Chong 1999). We have elected to describe the constitutive tissue behavior using Hooke's law of linear elasticity, which states that the strain is proportional to the applied stress, and further assume that materials are isotropic and nearly incompressible in nature. The description of the constitutive relationship between stress and strain is ultimately expressed in terms of the elasticity parameters $E$ (Young's modulus) and $v$ (Poisson's ratio).

A finite element representation of the model is constructed from the source image. Elements of the mesh are grouped using a K-means algorithm by initializing a number $(N)$ of seed points that are the centers of the clusters and iteratively minimizing their summed distance to all element centroids in the mesh. This process defines a set of nearly equally sized but spatially non-uniform regions that are homogeneous with respect to their material properties and establish the 'resolution' of the reconstructed elasticity image. After assigning appropriate boundary conditions based on estimated displacement or stress, the standard Galerkin method of weighted residuals (Lapidus and Pinder 1982) is used to construct a matrix system. The solution of that system yields displacements that are used to deform the source image. A second discretization is performed by binning the target image into $M$ groups of contiguous voxels termed zones. The model-deformed image is then compared to the target by summing 
the similarity metric evaluated for all zones. The correlation coefficient (Fitzpatrick et al 2000) is used throughout this work as it has empirically demonstrated better performance for our method over other intensity-based metrics such as the sum of squared differences and normalized mutual information. Optimization of the elasticity parameters is taken as the minimization of the objective function:

$$
\Psi=\left|S_{\text {TRUE }}-S_{\text {EST }}\right|^{2}
$$

where $S_{\text {TRUE }}$ is the set of similarity values achieved when comparing the target image to itself, $S_{\mathrm{EST}}$ is the similarity between the target and model-deformed source images using current estimates of the elastic modulus distribution and $|\bullet|$ denotes the vector $L^{2}$-norm. Note that by definition, $S_{\text {TRUE }}$ for the correlation coefficient has a constant value of 1. Differentiating (2) with respect to the elasticity distribution and setting the resulting expression equal to zero generates a series of nonlinear equations that can be solved using the Levenberg-Marquardt method:

$$
\begin{aligned}
& {\left[\mathbf{J}^{T} \mathbf{J}+\alpha I\right]\{\Delta E\}=\left[\mathbf{J}^{T}\right]\left\{S_{\mathrm{TRUE}}-S_{\mathrm{EST}}\right\}} \\
& \alpha^{2}=\lambda\left[\frac{1}{N} \sum_{i=1}^{N}\left(\mathbf{J}^{T} \mathbf{J}\right)_{i i}\right] \Psi^{2}
\end{aligned}
$$

where $\mathbf{J}$ is the Jacobian matrix of size $M \times N$ and $\Delta E$ is the vector of updates to the material property distribution defined by the regions. The regularization parameter $\alpha$ uses an empirical scalar factor $\lambda$ as determined by the methods described in Joachimowicz et al (1991). Each column of the Jacobian matrix is a finite difference approximation of the change in image similarity over all zones due to the perturbation of a single material property region, such that

$$
J \equiv \frac{\partial S_{\mathrm{EST}}}{\partial E}=\left[\begin{array}{cccc}
\frac{\partial S_{1}}{\partial E_{1}} & \frac{\partial S_{1}}{\partial E_{2}} & \cdots & \frac{\partial S_{1}}{\partial E_{N}} \\
\frac{\partial S_{2}}{\partial E_{1}} & \ddots & & \vdots \\
\vdots & & \ddots & \vdots \\
\frac{\partial S_{M}}{\partial E_{1}} & \cdots & \cdots & \frac{\partial S_{M}}{\partial E_{N}}
\end{array}\right]
$$

Modulus values contained in $E$ are updated by $\Delta E$ until an error tolerance on the relative objective function error evaluation is reached or a maximum number of iterations are completed. Spatial averaging of elasticity values in the model and solution relaxation between iterations are also utilized to improve the stability of the optimization.

\subsection{Parallel computing framework}

The transition of this method from 2D to 3D entails a much higher computational overhead that affects all parts of the reconstruction. The mesh needed to describe the entire breast as opposed to a single slice is at least 20-40 times greater in the number of structural components (nodes and elements), and the model must account for an additional degree of freedom. The resulting system of equations to be solved is thus nearly two orders of magnitude larger. The finite difference approximation of each column of the Jacobian matrix requires a 'forward solve' consisting the biomechanical model, image deformation and evaluation of the similarity metric. Because this must be done for every elasticity region, attempting to adequately sample the spatial domain makes the building of this matrix the primary expenditure of computing resources. 
In order to achieve a reasonable level of performance, the Message Passing Interface standard for parallel processing is used to distribute formation of the Jacobian among a number of communicating nodes controlled within a static single process, multiple data (SPMD) scheme. The Portable Extensible Toolkit for Scientific Computation (PETSc) (Balay et al 2004, 1997) has provided the necessary coding base for interfacing sparse matrix system solvers with our $\mathrm{C} / \mathrm{C}++$ Gauss-Newton optimization routine. This design scales readily to the number of processors available; it has been tested on a homogeneous cluster of 18 processors (2.0 GHz Pentium4 Xeon, 1 GB RAM) located in the laboratory, as well as a heterogeneous cluster of hundreds of processors available through the Vanderbilt Advanced Computing Center for Research and Education project. The use of many processors is capable of producing a nearly linear speedup and otherwise agrees in principle with the performance impact suggested by Ahmdahl's law (Ahmdahl 1967).

\subsection{Simulation experiment setup}

For this work, a simulation experiment is defined by the creation of an idealized target image volume from a deformation achieved by the specification of boundary conditions at the surface of the breast. This ensures data fidelity in order to effectively evaluate reconstruction performance in the optimization and model characteristics. Two image volumes of human breast were made available to further test the modality independence of the algorithm. The first was obtained from a dedicated breast CT scanner $\left(256 \times 256 \times 130\right.$, voxel size $\left.0.6 \mathrm{~mm}^{3}\right)$ as described in Boone et al (2006), (2001) and Boone and Lindfors (2006) and the second from a Philips Achieva 3.0 T MR unit $\left(256 \times 256 \times 98\right.$, voxel size $\left.1.0 \mathrm{~mm}^{3}\right)$ using a clinically approved transmit-receive double-breast coil to acquire a 3D T1-weighted exam with a fatnulling inversion pulse (TR/TE/a/NEX $\left.=4.6 \mathrm{~ms} / 2.3 \mathrm{~ms} / 10^{\circ} / 1\right)$ (Yankeelov et al 2007). The surfaces of the breast were segmented (ANALYZE 6.0, Mayo Clinic, Rochester, MN) to create tetrahedral meshes composed of 39013 nodes connected in 214163 elements for the CT volume and 20623 nodes and 111142 elements for the MR volume. A $2 \mathrm{~cm}$ spherical tumor was synthetically implanted in the center of each mesh by assigning a stiff modulus to appropriate member elements that was six times higher than the surrounding material (Krouskop et al 1998, Samani et al 2007). Tissue deformation was performed by creating a set of displacements calculated to approximate a Gaussian stress distribution applied to a rectangular area on the lateral surface of the breast. The displacements were then applied to the original volumes in order to create the desired target images. Figure 2 illustrates the setup of the simulation data.

\subsection{Reconstruction experiments}

Reconstructions using spatial a priori knowledge of the location and size of the inclusion were first performed in order to constrain the problem, as well as the computational expense of the Jacobian matrix, to a two-material discrimination of relative stiffness (elastic contrast). A second set of experiments was then used to address the viability of the method to perform a generalized detection of the lesion with no knowledge of the actual structure of the domain. To run these naive reconstructions for the CT data set, 3180 material regions and 733 voxel similarity zones were partitioned, while in the MR data set, 3166 regions and 768 zones were used. In all cases, the reconstruction was initialized with a homogeneous elasticity distribution, and the value of Poisson's ratio held constant at $v=0.485$ to represent a nearly incompressible material. 

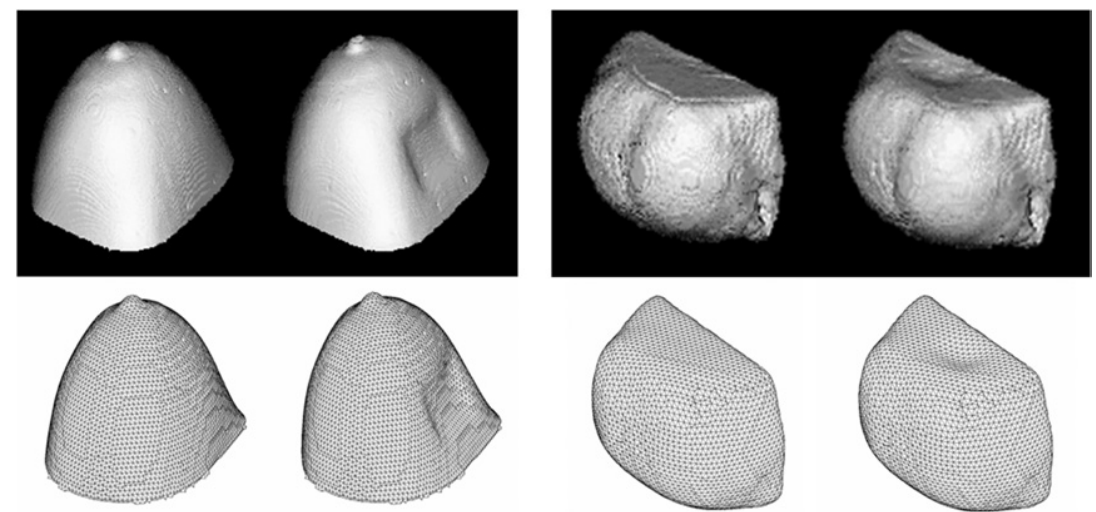

(a)

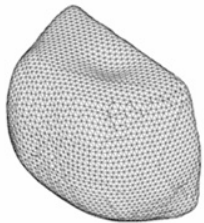

(b)

Figure 2. (a) CT data set and (b) MR data set used for 3D MIE simulations. Surface renderings of the image volumes (top row) and meshes (bottom row) are shown for the pre- (source) and post-deformation (target) scenarios.

\subsection{Evaluating boundary condition influence}

In addition to image acquisition, the other major input to the reconstruction algorithm is the delineation of boundary conditions on the region of interest over which the model is applied. While relatively easy to control in simulation, in a real clinical situation, this presents the challenge of accurately determining point correspondences between the source and target breast surfaces. The effect of any inaccuracies is cumulative, as errors are propagated from the model to the image deformation and finally the similarity measurements. In previous $2 \mathrm{D}$ work, manual delineation of boundary conditions was possible with guidance and correction using standard computer input devices (i.e. a mouse). However, the increased complexity of mesh geometry in 3D necessitates a more automated technique of determining correspondence between two surfaces. Potentially non-trivial random and/or operator-dependent noise is introduced into any generated boundary conditions. Therefore, the following experiments were performed to examine the ability of the algorithm to tolerate various types of mismappings.

2.5.1. Robustness to randomized boundary errors. The gold standard boundary conditions used to create the simulated target image volumes were deliberately disrupted to examine the effect of random noise on reconstruction fidelity. A series of magnitudes ranging from 0.01 to 2.0 voxel units (mesh coordinates normalized by their respective spacing in image space) were applied to the CT and MR data sets. Therefore, every boundary position is displaced by the same amount but in a completely unpredicted manner, as illustrated in figure 3 . These altered boundary conditions sets were utilized in the reconstruction of the a priori two-material test case, and the tolerance of the method was evaluated by calculating the average reconstructed elasticity contrast ratios over four trials of each level of noise, with deviations less than $20 \%$ from the true stiffness being deemed acceptable.

2.5.2. Feasibility of automated boundary condition generating methods. Three methods of surface registration and point correspondence were considered as the basis of a semi-automated method for determining boundary conditions input to the reconstruction algorithm. Two were 


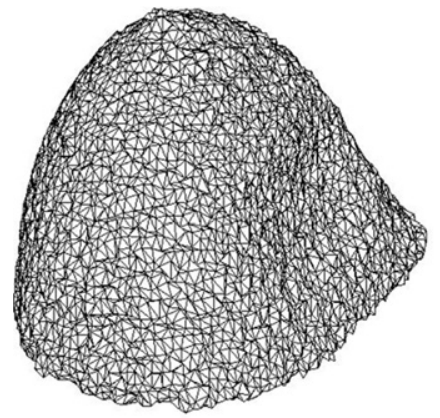

(a)

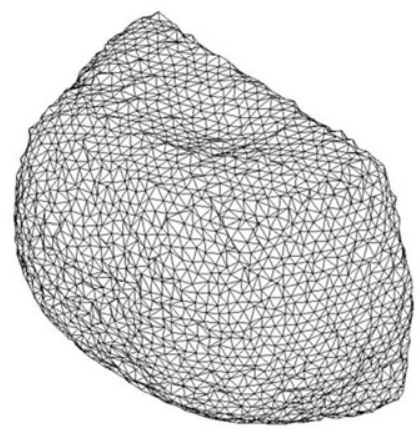

(b)

Figure 3. Examples of distortion due to additive randomized error. For effect, noise of 2.0 voxel units is shown as applied to the gold standard boundary condition set for CT (a) and MR (b). At these extreme levels, the smooth surface of the breast as originally captured in figure 2 is completely lost, and the forced reconfiguration of internal elements in the finite element mesh adversely affects all aspects of the reconstruction.

specifically developed for this work by attempting to use potential energy distributions derived from classic PDEs for surface matching, and the other is a free-form geometrical warping.

If the flow of a hypothetical substance over both the source and target breast surfaces is taken to be a conserved process and modeled using potential theory, correspondence can be assigned by matching areas of similar energy deposition, that is, the equivalent level sets. The algorithm for the PDE-based surface matching methods can be summarized in the following steps:

(1) Determine an energy distribution for each surface. Laplace's equation is commonly used to describe the steady-state distribution of potential energy $\Phi$ in a system:

$$
-\nabla^{2} \Phi=0 \text {. }
$$

Similarly, the diffusion equation describes the temporal change in potential over a region:

$$
\frac{\partial \Phi}{\partial t}=D \nabla^{2} \Phi
$$

where $D$ is the diffusion coefficient. Each PDE is solved over both breast surfaces (source and target). For both equation (5) and (6), the nipple is assigned as an area of high potential energy. Additionally, with equation (5), nodes at the chest wall are assigned a value of 0 in order to obtain a non-trivial solution, whereas the propagating front produced by (6) is artificially halted at the chest wall boundary. While both PDE solutions similarly establish an energy gradient over the breast surfaces, their application in the following steps results in more apparent differences.

(2) Determine correspondence between energy distributions. From the solution of the PDEs on the source surface, a series of spatially distributed isocontours representing distinct potentials are determined. For each level set, an isocontour of equivalent potential energy is found on the target surface and the two curves matched according to the symmetric closest point method described by Papademetris et al (2002).

(3) Generate boundary conditions. By extracting a number of isocontours of different values, the resulting point correspondence vectors define a relatively dense $3 \mathrm{D}$ displacement field. The displacement for each boundary node can then be interpolated from the set of its nearest neighbors. 
The final method employs thin-plate splines (Goshtasby 1988) to generate a set of boundary conditions. In this well-established method of non-rigid transformation, a number of control points with known correspondences establish constraints on the deflection of a hypothetical thin sheet of material in order to best warp the two surfaces together. Displacements at each boundary node are then simply interpolated from the calculated fit. For these simulation experiments, a subset of boundary nodes was used to represent physical markers on the breast surface. Forty points were uniformly distributed over the CT mesh and eighty for the MR mesh in order to handle the more highly variegated shape of the latter data set.

The automated methods were initially evaluated according to their target registration error (TRE), which was calculated as the average Euclidean distance between the generated and true boundary conditions. Because the deployment of these fits represents a more correlated form of noise, these boundary conditions were also applied to the two-material (a priori) scenario and the reconstructed elasticity contrast values compared to the trials of additive randomized error for which the magnitude was approximately equal to the TRE. Finally, a mapping of the objective function space was performed by calculating the similarity values for model-based image deformations over a range of elasticity contrasts from 0.5:1 to 30:1. An interpolating curve was fit to extract the minimum objective function value and associated contrast ratio to determine a theoretical optimal reconstruction as constrained by the estimated boundary conditions.

\section{Results}

\subsection{MIE reconstructions}

Because the use of a priori spatial information about the inclusion limits the reconstruction to a two-material system, the fidelity of the reconstruction is simply evaluated by examining the elastic contrast between the inclusion and the normal tissue of the breast (ideal of 6:1). Figure 4 demonstrates the behavior of the algorithm in optimizing the objective function while successfully characterizing the stiffness of the inclusion to within 5\% of the actual value (6.02:1 and 6.21:1 for the CT and MR data sets, respectively).

The fidelity of the generalized reconstruction experiments (using no a priori knowledge of the domain) was primarily evaluated on its ability to detect the presence of an inclusion based on classification of the material property distribution as well as the retrospective accuracy of localizing the lesion. The final elasticity values were treated as a Gaussian mixture of two classes and separated by a threshold established via the method described in Otsu (1979). The likelihood of discriminating a lesion in the resulting elasticity image was found using the contrast-to-noise ratio (CNR) as defined by Bilgen (1999) and Doyley et al (2003):

$$
\mathrm{CNR}=\sqrt{\frac{2\left(\mu_{L}-\mu_{B}\right)^{2}}{\sigma_{L}^{2}+\sigma_{B}^{2}}}
$$

where $\mu$ and $\sigma^{2}$ are the sample mean and variance of a material property distribution and the subscripts $L$ and $B$ denote the lesion and normal material types, respectively. As a quantitative assessment of the localization of the lesion, the positive predictive value of correctly identifying a lesion material within the known segmented region of the inclusions was also calculated as a 'quality of reconstruction score' (QRS). This value is significant because identification of the lesion border and material classification are done independently, so user knowledge of the test scenario does not influence the performance of the measure. The 'true positive' (TP) elements of the mesh are counted as the number correctly identified as tumor and lying within the 

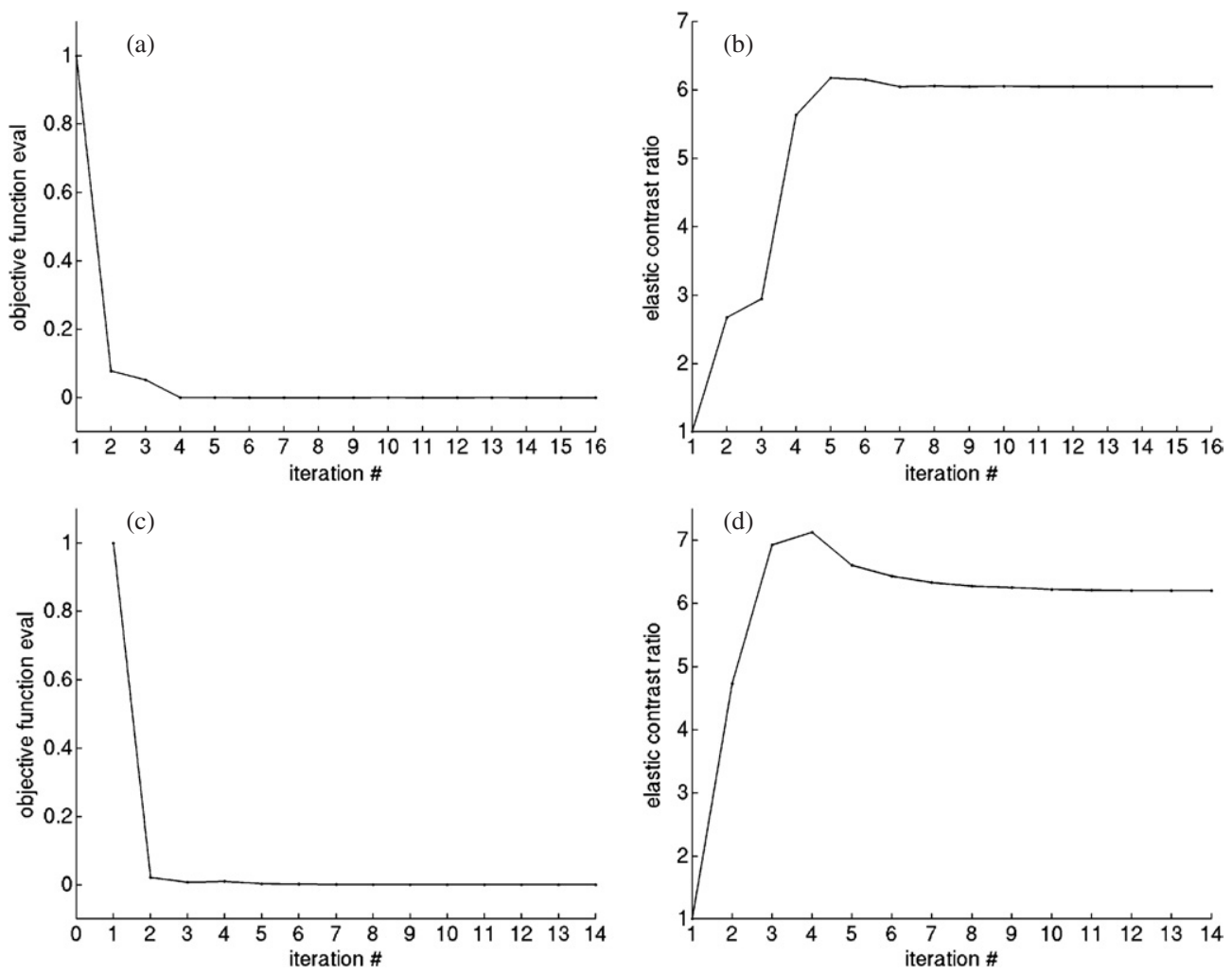

Figure 4. Optimization behavior of reconstructions using a priori knowledge of the inclusion location. For the CT simulation, the objective function evaluation (normalized to the initial dissimilarity value of a homogeneous elasticity distribution) and elastic contrast over several iterations of the algorithm are shown in panels (a) and (b), respectively. Similarly, this behavior for the MR data set is displayed in (c) and (d). In each case, the minimum value is achieved quickly and stably, with the corresponding contrast ratio matching the true value of 6:1 very closely (6.02:1 and 6.21:1 for CT and MR, respectively).

Table 1. Evaluation of reconstruction fidelity for lesion detection.

\begin{tabular}{lllll}
\hline & CNR & QRS $(\%)$ & Max CR $(\times: 1)$ & Optimal CR $(\times: 1)$ \\
\hline CT & 3.55 & 99.4 & 2.66 & 3.01 \\
MR & 3.93 & 99.7 & 2.02 & 2.26 \\
\hline
\end{tabular}

Max CR: maximum elasticity contrast between lesion and normal tissue in naive reconstruction. Optimal CR: optimal elasticity contrast after accounting for overlap in elasticity region partitioning.

known segmentation of the lesion, while the 'false positive' (FP) elements are those identified as tumor but in an incorrect location. Thus, the calculation of QRS is simply TP/(TP + FP). Cutoffs for successful detection and localization were set at CNR $\geqslant 2.2$ as noted by Doyley et al (2003) and QRS $\geqslant 80 \%$ as empirically determined by a prior study in 2D MIE work (Ou et al 2006a, 2006b), and both the CT and MR reconstructions successfully identified the embedded lesions according to these criteria (see table 1).

The peak modulus contrast value of a reconstruction was calculated by taking the ratio of the average elasticity for manually selected homogeneous regions of the approximately equal area known to be representative of the two materials. As reported in table 1 and visualized 
Table 2. Effect of applied random boundary condition noise on objective function space and reconstructed elasticity contrast ratio. The respective ranges where a cutoff in reconstruction tolerance was observed are listed for each simulation set.

\begin{tabular}{lllll}
\hline \multicolumn{2}{c}{ CT } & & \multicolumn{2}{c}{ MR } \\
\cline { 1 - 2 } \cline { 5 - 5 } $\begin{array}{l}\text { Randomized } \\
\text { vector magnitude } \\
\text { (voxel units) }\end{array}$ & $\begin{array}{l}\text { Mean optimal } \\
\text { elasticity contrast } \\
\text { value }(\times: 1)\end{array}$ & & $\begin{array}{l}\text { Randomized } \\
\text { vector magnitude } \\
\text { (voxel units) }\end{array}$ & $\begin{array}{l}\text { Mean optimal } \\
\text { elasticity contrast } \\
\text { value }(\times: 1)\end{array}$ \\
\hline 0.1 & $5.62 \pm 0.421$ & & 0.01 & $6.33 \pm 0.096$ \\
0.2 & $5.70 \pm 0.588$ & & 0.02 & $6.75 \pm 0.058$ \\
0.3 & $5.97 \pm 0.846$ & & 0.03 & $6.93 \pm 0.634$ \\
0.5 & $2.36 \pm 0.393$ & & 0.05 & $7.60 \pm 0.821$ \\
1.0 & $2.47 \pm 0.266$ & & 0.1 & $9.35 \pm 1.27$ \\
2.0 & $2.17 \pm 0.422$ & & 0.2 & $11.3 \pm 0.866$ \\
\hline
\end{tabular}

Table 3. Reconstruction performance as affected by semi-automated boundary condition generation methods. The mean error of surface registration is related to the accuracy of characterizing the lesion stiffness.

\begin{tabular}{llllll}
\hline & \multicolumn{2}{c}{ CT } & & \multicolumn{2}{c}{ MR } \\
\cline { 2 - 3 } \cline { 5 - 6 } Method & $\begin{array}{l}\text { TRE } \\
(\mathrm{mm})\end{array}$ & $\begin{array}{l}\text { Elasticity } \\
\text { contrast }(\times: 1)\end{array}$ & & $\begin{array}{l}\text { TRE } \\
(\mathrm{mm})\end{array}$ & $\begin{array}{l}\text { Elasticity } \\
\text { contrast }(\times: 1)\end{array}$ \\
\hline Diffusion & 1.5 & 17.5 & & 0.61 & 348 \\
Laplace & 0.52 & 5.02 & & 0.48 & 673 \\
Thin-plate spline & 0.26 & 5.66 & & 0.023 & 6.26 \\
\hline
\end{tabular}

in figures 5 and 6 , the characterization of the relative stiffness is less than the true elasticity contrast by nearly a factor of 3 in both cases. This reveals a difficulty with large inverse problems in 3D where a need for reasonable performance can lead to a tradeoff in accuracy. By choosing approximately 3200 regions to cover the domain of the breasts for the naive reconstructions, the number of degrees of freedom presented to the optimization scheme is quite high. However, this is also relatively coarse in the sense of visualizing the reconstruction, as it roughly corresponds to a $15 \times 15 \times 15$ image volume. Because the elasticity regions do not conform perfectly to the actual lesion borders and furthermore comprise both the tumor and healthy tissue, it seemed reasonable to surmise that in this mis-estimation of spatial extents the algorithm was forced to attempt a best-fit compromise. To test this hypothesis, we agglomerated all regions in the original partitioning that overlapped the tumor and then ran the reconstruction again as a two-material characterization. Upon inspection, this re-grouping was clearly a larger entity than the tumor itself (closer to $3 \mathrm{~cm}$ in diameter) and resulted in a shift of the global optimum to a lower elasticity contrast. In effect, the model reacted to this new, oversized tumor by reducing its stiffness in order to achieve the proper image similarity match. When viewed in light of this analysis as summarized in table 1, the elasticity contrast found by the naive reconstruction is then actually quite accurate.

\subsection{Evaluating boundary condition influence}

3.2.1. Robustness to randomized noise. Table 2 demonstrates that as the magnitude of the applied randomized noise vectors was increased, changes in the reconstructed elasticity contrast reflected a decreased ability to achieve a successful result (recall that the correct ratio 


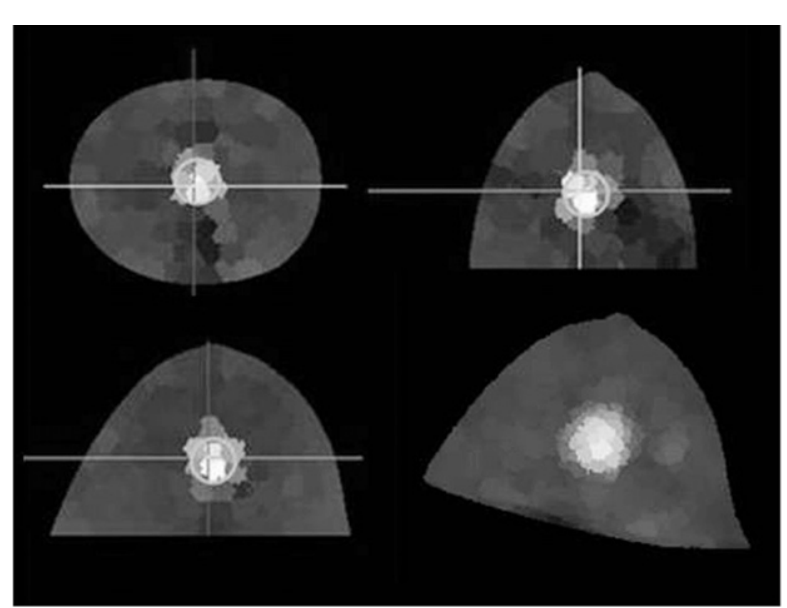

(a)
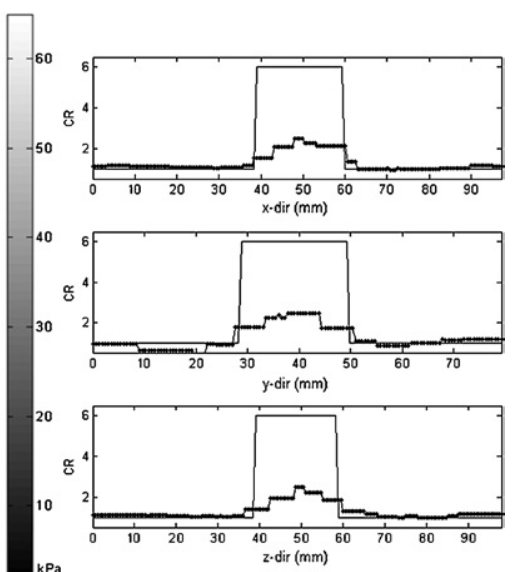

(b)

Figure 5. Reconstruction used for lesion detection in the CT data set. (a) Orthogonal views taken through the center of the elasticity image volume are shown along with a projection surface rendering (lower right). The simulated inclusion implanted in the mesh is visually distinguished from the surrounding tissue. The color bar indicates the range of elasticity values $(\sim 7-42 \mathrm{kPa})$ designated by the reconstruction, with higher (stiffer) values shown in the white end of the grayscale mapping. (b) Transect plots through the center of the volume along the cardinal directions show the profile of elasticity contrast (dotted lines) overlaid by the true profile of the simulation (solid lines).

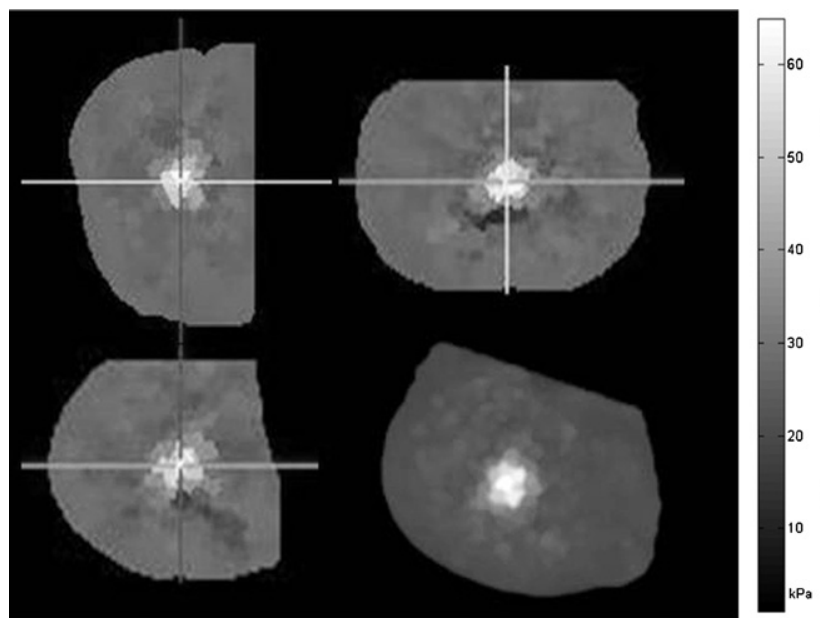

(a)
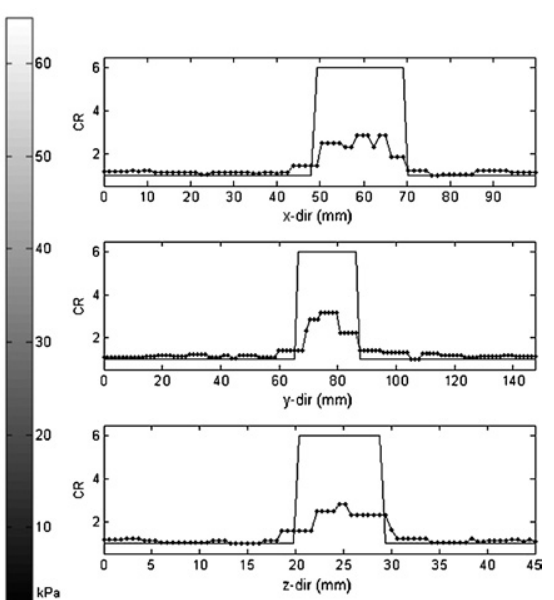

(b)

Figure 6. Reconstruction used for lesion detection in the MR data set. (a) Orthogonal views taken through the center of the elasticity image volume are shown along with a projection surface rendering (lower right). Once again, the inclusion appears to have a recognizably different elasticity, with values on the color bar ranging from $\sim 10-57 \mathrm{kPa}$. (b) Transect plots through the center of the volume along the cardinal directions show the profile of elasticity contrast (dotted lines) overlaid by the true profile of the simulation (solid lines).

is 6:1). For the CT simulation, on average, errors of 0.5 voxel units or greater showed a dramatically reduced ability to accurately characterize the stiffness of the lesion. Similarly, though at a much smaller scale, the MR simulation began to have noticeable difficulty in 

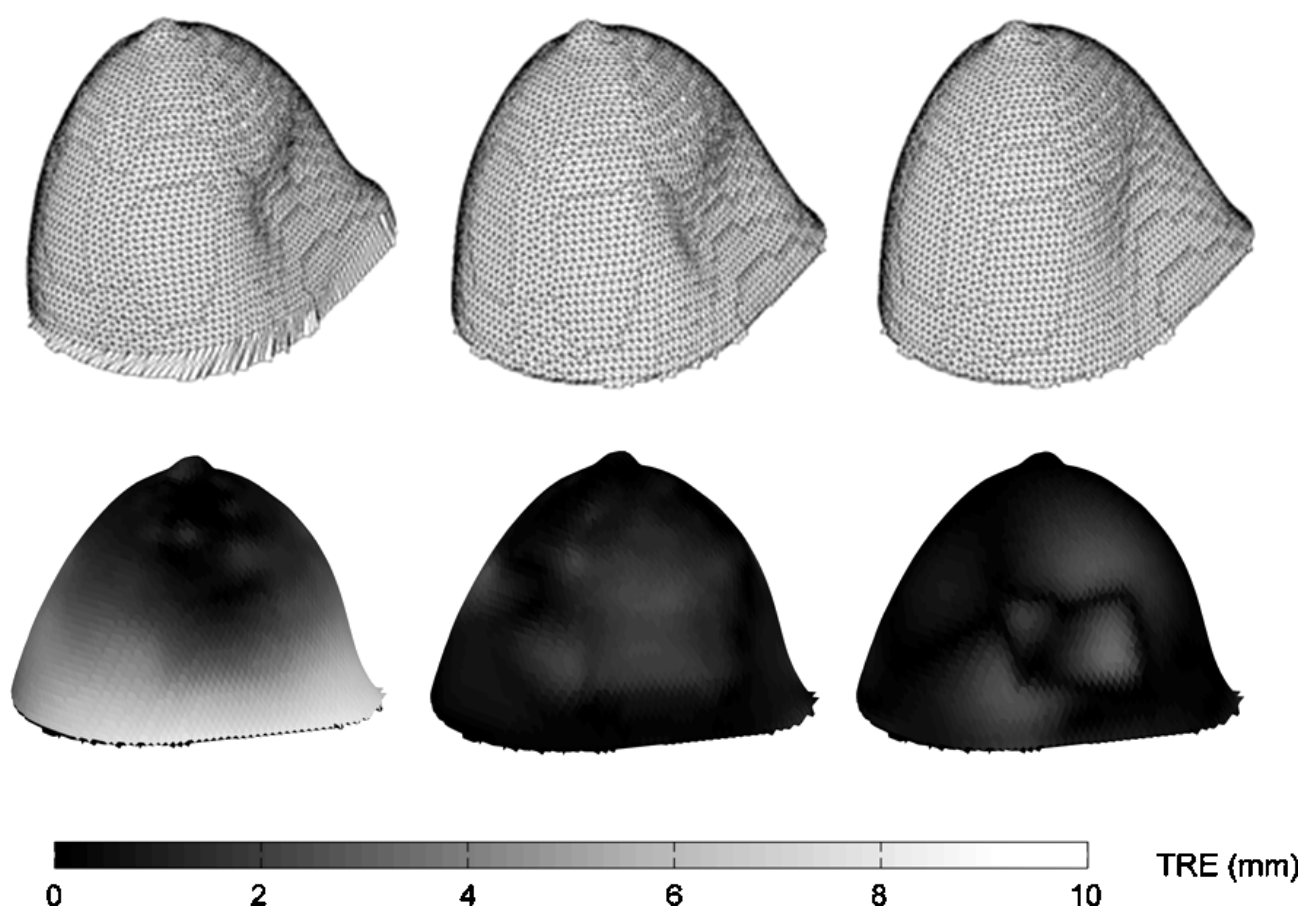

Figure 7. Three candidate automated methods for MIE boundary condition generation applied to simulation CT data. Top row, from left to right: surface deformations calculated from diffusion energy matching, Laplace solution energy and thin-plate spline interpolation. Bottom row: target registration error (TRE) distribution for each method when compared against the gold standard of known correspondence. The diffusion-based mesh is both qualitatively and quantitatively the worst performer. The Laplace solution appears to capture the shape of the bladder indentation more precisely, but the thin-plate spline has the best overall accuracy in determining point correspondence.

achieving a reasonable reconstruction at noise levels of 0.05 voxel units. These values were taken as suitably conservative measures for evaluating the efficacy of boundary conditions generated by the semi-automated methods.

3.2.2. Reconstruction effects of generated boundary conditions. The accuracy of each automated boundary condition technique described in section 2.5.2 was assessed by the TRE with respect to the gold standard boundary condition set and its ability to characterize the elastic contrast in the two-material reconstruction test case. Figure 7 depicts the deformation fields as applied to the CT data. Qualitatively, the displacements found by the diffusion method are quite different from the true set, while the results from the solution of Laplace's equation and the thin-plate spline interpolation appear to be more satisfactory. As shown in table 3, the mean TRE of the three methods confirms that the spline-based method has the best performance $(0.26 \mathrm{~mm})$, the Laplace method next $(0.52 \mathrm{~mm})$ and the diffusion method being the worst $(1.5 \mathrm{~mm})$. Inspection of figures 8 and 9 further demonstrates that the imposition of an inexact boundary condition set on the model has a distinct effect on the optimization by shifting the objective function minimum value to a different optimal elastic contrast ratio. Additionally, the convexity of the objective function is lost in the cases with a higher TRE. The differences in the generated boundary condition sets for the MR simulation are not easily visualized but follow a similar performance trend (TRE of spline $0.023 \mathrm{~mm}$, Laplace method 

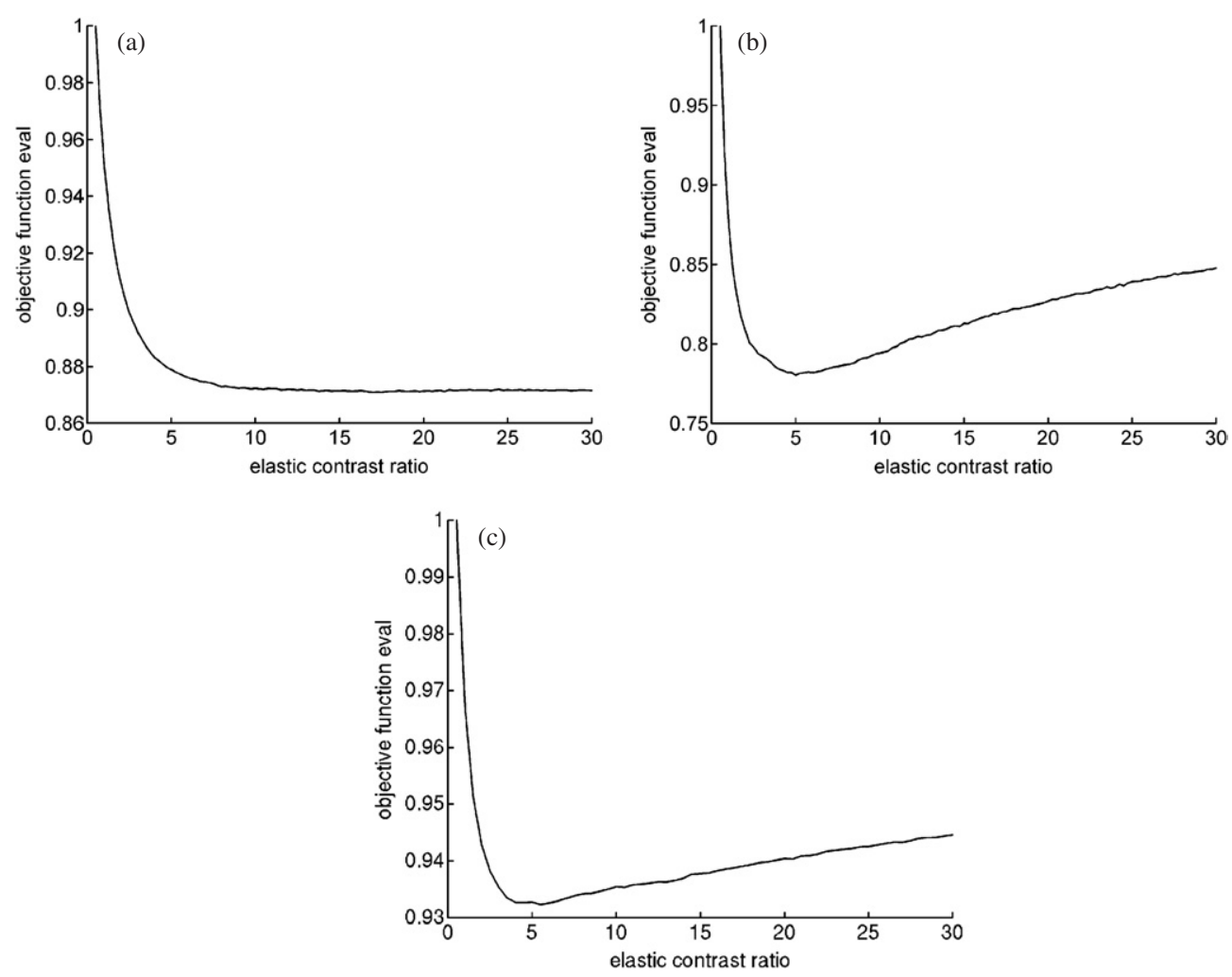

Figure 8. Mappings of objective function value versus elasticity contrast ratio (tumor:breast) affected by the boundary condition sets generated from the different automated methods of surface point correspondence as applied to the CT data set. The minimum value of each curve corresponds to the altered optimal elasticity contrast when constrained by the inaccuracies of the methods: (a) diffusion, (b) Laplace and (c) thin-plate spline interpolations. The ordinate is normalized to the initial value of each case. The global minimum of (a) is out of range of the plot.

$0.48 \mathrm{~mm}$, diffusion $0.61 \mathrm{~mm}$ ). For both simulations, there exists a direct relationship between a low TRE and increased reconstruction fidelity in characterization of the elasticity contrast of the lesion.

\section{Discussion}

As other researchers have noted, the incorporation of a priori information can greatly enhance the performance of their elastography methods (Doyley et al 2005, 2006). We recognize that the judicious use of information regarding lesion morphology as obtained from conjunctive imaging studies and post-processing would potentially aid MIE as well, especially in reducing the number of search parameters and improving initialization of the algorithm. The reconstructions using a priori spatial knowledge of the inclusion were initially intended to simply illustrate that the objective function space formed by using an image similarity metric was smooth and readily traversed by the algorithm in a manner expected for a Gauss-Newton optimization. However, they also provide a stark contrast to the naive lesion detection test cases, which were performed to evaluate the inverse problem framework and demonstrate its ability to analyze the full 3D domain of the breast. The results of the generalized reconstructions are very encouraging in having successfully identified 

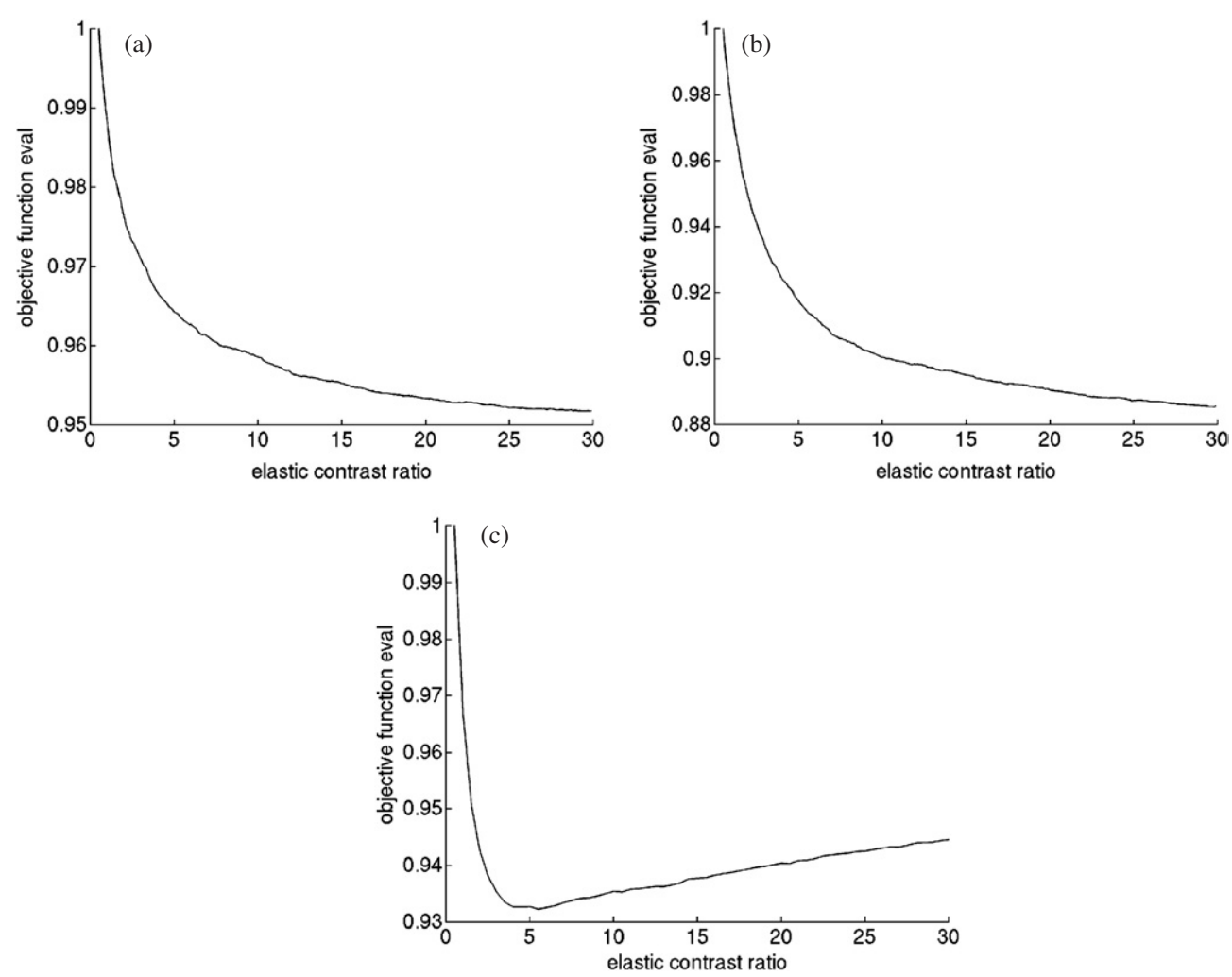

Figure 9. Mappings of objective function value versus elasticity contrast ratio (tumor:breast) affected by the boundary condition sets generated from the different automated methods of surface point correspondence as applied to the MR data set. The minimum value of each curve corresponds to the altered optimal elasticity contrast when constrained by the inaccuracies of the methods: (a) diffusion, (b) Laplace and (c) thin-plate spline interpolations. Again, the ordinate is normalized to the initial value and should not be interpreted as an equivalent scale for each case. The global minima of (a) and (b) are out of range of the plot.

and localized the inclusions. Although the discretizations of the meshes did not achieve particularly accurate material characterizations, the optimal elasticity contrast as dictated by the available objective function was matched in each case to within $12 \%$. The observation that mis-estimation of the lesion extent altered the underlying test scenarios suggests that investigating methods of dynamically adjusting region assignment could facilitate shape resolution and concomitantly better elasticity contrast ratio values.

In translating MIE and its associated technologies to a clinical setting, a number of factors must be considered for realistic deployment. From an implementation and performance perspective, the large size of the inverse problem necessitated the careful selection of matrix solvers and programming of parallel computing routines that proved effective with the availability of a number of processors. Initial predictions based on sequential execution times needed to handle the high degrees of freedom in the naive reconstructions were thus reduced from two weeks to several hours. Additional challenges were eventually overcome in the pre-processing load of image segmentation, model generation and partitioning schemes.

The results presented in this paper also further our understanding of how the loss of input data quality, whether through design limitations or unpredictable factors, could have a significant impact on the end reconstruction. In particular, the proper application of accurate 
boundary conditions plays a critical role in MIE reconstruction success. This is due to the link between surface shape matching and subsequent interpolation of internal displacements in affecting sub-surface image intensities and similarity measurements. The results of the boundary condition noise experiment are interesting because they indicate that some level of improper localization of surface point correspondence is reasonably tolerated by the algorithm. However, perturbations greater than an empirically observed threshold can impair its ability to determine the underlying elasticity distribution. This is a similar result to prior work done in 2D systems for which successful reconstructions correlated to boundary condition selection errors limited to half a pixel length (Ou et al 2006b). It also confirms that randomizing the vectors for the additive noise experiments poses a considerable challenge to the algorithm because of the introduction of grossly non-physical deformations in the finite element mesh that decrease the stability of the numerical model. We observed that the threshold for the MR simulation was an order of magnitude less than that of the CT set and initially seemed to require an unfeasible level of accuracy, as well as quite a few more fiducials. These key differences are likely related to image resolution (the MR volume had fewer slices and a larger voxel spacing) and to the inherent differences in soft-tissue contrast between the two modalities. Both issues present interesting questions that will be explored in future work.

The implausibility of performing manual selection on all boundary nodes of a 3D mesh (there were 6319 points for the CT and 5416 for the MR set) underscores the importance of finding an automated method for determining point correspondences. In general, energy matching from the solutions of the diffusion and Laplace equations yielded boundary condition sets that were inadequate for reconstructing a proper elasticity contrast. This can be partly explained because the TRE of those surface registration techniques (as compared to the gold standard) was typically greater than the permissible value established by the robustness tests. The primary manifestation of these poor matches was that the model often had difficulty in obtaining a stable solution. Indeed, only the boundary conditions generated by thin-plate spline method, which had the least error, were able to consistently achieve successful reconstructions while also satisfying the putative cutoffs. Overall, the reconstruction behavior for this method was consistent to within $6 \%$ of the true value. This appears to recommend the use of thin-plate spline interpolation as a strong candidate for generating boundary conditions for MIE.

\section{Conclusions}

In this work, we have presented the first fully 3D realization of the MIE algorithm and preliminary evaluation of accompanying strategies for automated boundary condition deployment. The use of parallel processing enabled a practical implementation of a computational problem that might otherwise prove intractable. Simulation experiments demonstrate the viability of the method to utilize images obtained from different sources in reconstructing an embedded lesion with or without the benefit of a priori information concerning its location and size. We have also characterized the robustness of the elastography method to inaccuracies in boundary condition inputs derived from either random noise or by surface point correspondence methods. These results should prove valuable in the customization and streamlining of data acquisition and pre-processing for forthcoming clinical tests.

\section{Acknowledgments}

The authors would like to acknowledge John Boone, $\mathrm{PhD}$ (University of California-Davis Medical Center, Department of Radiology), for the donation of the CT data set, and Prashanth 
Dumpuri, PhD (Vanderbilt University, Department of Biomedical Engineering), for software development support. Additional thanks goes to the Vanderbilt Advanced Computing Center for Research and Education for the use of cluster resources. This work was funded by a Breast Cancer Research Program Predoctoral Traineeship Award (BC043661) of the Congressionally Directed Medical Research Program along with a Whitaker Foundation Young Investigator Award. TE Yankeelov is supported by NIBIB 1K25EB005936-01.

\section{References}

ACS 2007 Cancer Facts and Figures (Atlanta: American Cancer Society)

Ahmdahl G 1967 Validity of the single processor approach to achieving large-scale computing capabilities AFIPS Conf. Proc. pp 483-5

Balay S, Buschelman K, Eijkhout V, Gropp W, Kaushik D, Knepley M, McInnes L, Smith B and Zhang H 2004 PETSc Users Manual (Argonne, IL: Argonne National Laboratory)

Balay S, Gropp W D, McInnes L C and Smith B F 1997 Modern Software Tools in Scientific Computing (Basle: Birkhauser) pp 163-202

Bilgen M 1999 Target detectability in acoustic elastography IEEE Trans. Ultrason. Ferroelectr. Freq. Control $461128-33$

Boone J M, Kwan A L, Yang K, Burkett G W, Lindfors K K and Nelson T R 2006 Computed tomography for imaging the breast J. Mammary Gland Biol. Neoplasia 11 103-11

Boone J M and Lindfors K K 2006 Breast CT: potential for breast cancer screening and diagnosis Future Oncol. (London, England) 2 351-6

Boone J M, Nelson T R, Lindfors K K and Seibert J A 2001 Dedicated breast CT: radiation dose and image quality evaluation Radiology $221657-67$

Boresi A and Chong K P 1999 Elasticity in Engineering Mechanics (New York: Wiley-Interscience)

Curiel L, Souchon R, Rouviere O, Gelet A and Chapelon J Y 2005 Elastography for the follow-up of high-intensity focused ultrasound prostate cancer treatment: initial comparison with MRI Ultrasound Med. Biol. 31 1461-8

Doyley M M, Srinivasan S, Dimidenko E, Soni N and Ophir J 2006 Enhancing the performance of model-based elastography by incorporating additional a priori information in the modulus image reconstruction process Phys. Med. Biol. 51 95-112

Doyley M M, Srinivasan S, Pendergrass S A, Wu Z and Ophir J 2005 Comparative evaluation of strain-based and model-based modulus elastography Ultrasound Med. Biol. 31 787-802

Doyley M M, Weaver J B, Van Houten E E, Kennedy F E and Paulsen K D 2003 Thresholds for detecting and characterizing focal lesions using steady-state MR elastography Med. Phys. 30 495-504

Egorov V, Ayrapetyan S and Sarvazyan A P 2006 Prostate mechanical imaging: 3-D image composition and feature calculations IEEE Trans. Med. Imaging 25 1329-40

Fitzpatrick J M, Hill D L G and Maurer C R 2000 Handbook of Medical Imaging (Bellingham, WA: SPIE Optical Engineering Press) pp 447-513

Fowlkes J B, Emelianov S Y, Pipe J G, Skovoroda A R, Carson P L, Adler R S and Sarvazyan A P 1995 Magneticresonance imaging techniques for detection of elasticity variation Med. Phys. 22 1771-8

Garra B S, Cespedes E I, Ophir J, Spratt S R, Zuurbier R A, Magnant C M and Pennanen M F 1997 Elastography of breast lesions: initial clinical results Radiology 202 79-86

Gokhale N, Richards M, Oberai A, Barbone P E and Doyley M M 2004 Simultaneous elastic image registration and elastic modulus reconstruction IEEE Int. Symp. on Biomedical Imaging (Arlington, VA: IEEE Press) pp 543-6

Goshtasby A 1988 Registration of images with geometric distortions IEEE Trans. Geosci. Remote Sens. 26 60-4

Joachimowicz N, Pichot C and Hugonin J P 1991 Inverse scattering: an iterative numerical method for electromagnetic imaging IEEE Trans. Biomed. Eng. 39 1742-52

Krouskop T A, Wheeler T M, Kallel F, Garra B S and Hall T 1998 Elastic moduli of breast and prostate tissues under compression Ultrason. Imaging 20 260-74

Lapidus L and Pinder G F 1982 Numerical Solution of Partial Differential Equations in Science and Engineering (New York: Wiley)

Manduca A, Oliphant T E, Dresner M A, Mahowald J L, Kruse S A, Amromin E, Felmlee J P, Greenleaf J F and Ehman R L 2001 Magnetic resonance elastography: non-invasive mapping of tissue elasticity Med. Image Anal. $5237-54$

McKnight A L, Kugel J L, Rossman P J, Manduca A, Hartmann L C and Ehman R L 2002 MR elastography of breast cancer: preliminary results AJR Am. J. Roentgenol. 178 1411-7 
Melodelima D, Bamber J C, Duck F A, Shipley J A and Xu L 2006 Elastography for breast cancer diagnosis using radiation force: system development and performance evaluation Ultrasound Med. Biol. 32 387-96

Miga M I 2002 A new approach to elastographic imaging: modality independent elastography Med. Imag. 2002: Image Processing (San Diego, CA: SPIE Press) pp 604-11

Miga M I 2003 A new approach to elastography using mutual information and finite elements Phys. Med. Biol. 48 467-80

Miga M I, Rothney M P and Ou J J 2005 Modality independent elastography (MIE): potential applications in dermoscopy Med. Phys. 32 1308-20

Muthupillai R, Lomas D J, Rossman P J, Greenleaf J F, Manduca A and Ehman R L 1995 Magnetic resonance elastography by direct visualization of propagating acoustic strain waves Science 269 1854-7

Ophir J, Cespedes I, Ponnekanti H, Yazdi Y and Li X 1991 Elastography: a quantitative method for imaging the elasticity of biological tissues Ultrason. Imaging 13 111-34

Ophir J, Garra B, Kallel F, Konofagou E, Krouskop T, Righetti R and Varghese T 2000 Elastographic imaging Ultrasound Med. Biol. 26 (Suppl 1) S23-9

Otsu N 1979 Threshold selection method from gray-level histograms IEEE Trans. Syst. Man Cybern. 9 62-6

Ou J J, Barnes S L and Miga M I 2006a Application of multi-resolution modality independent elastography for detection of multiple anomalous objects Medical Imaging 2006: Physiology, Function and Structure from Medical Images (San Diego, CA: SPIE Press) pp 614310-1-9

Ou J J, Barnes S L and Miga M I 2006b Preliminary testing of sensitivity to input data quality in an elastographic reconstruction method IEEE Int. Symp. Biomed. Imaging (Arlington, VA: IEEE Press) pp 948-51

Papademetris X, Sinusas A J, Dione D P, Constable R T and Duncan J S 2002 Estimation of 3-D left ventricular deformation from medical images using biomechanical models IEEE Trans. Med. Imaging 21 786-800

Parker K J, Taylor L S, Gracewski S and Rubens D J 2005 A unified view of imaging the elastic properties of tissue J. Acoust. Soc. Am. 117 2705-12

Samani A, Zubovits J and Plewes D 2007 Elastic moduli of normal and pathological human breast tissues: an inversion-technique-based investigation of 169 samples Phys. Med. Biol. 52 1565-76

Sarvazyan A P, Skovoroda A R, Emelianov S Y, Fowlkes J B, Pipe J G, Adler R S, Buxton R B and Carson P L 1995 Acoustical Imaging ed J P Jones (New York: Plenum) pp 223-40

Sinkus R, Lorenzen J, Schrader D, Lorenzen M, Dargatz M and Holz D 2000 High-resolution tensor MR elastography for breast tumour detection Phys. Med. Biol. 45 1649-64

Tsap L V, Goldgof D B, Sarkar S and Powers P S 1998 A vision-based technique for objective assessment of burn scars IEEE Trans. Med. Imaging 17 620-33

Washington C W and Miga M I 2004 Modality independent elastography (MIE): a new approach to elasticity imaging IEEE Trans. Med. Imaging 23 1117-28

Yankeelov T E et al 2007 Integration of quantitative DCE-MRI and ADC mapping to monitor treatment response in human breast cancer: initial results Magn. Reson. Imaging 25 1-13

Zhang Y, Goldgof D B and Sarkar S 2004 Significance of elastic properties in physics-based nonrigid motion modeling: a numerical sensitivity analysis $C V P R W$ p 21 